\title{
Adenovirus-associated Acute Respiratory Distress Syndrome: Need for a Protocol-based Approach
}

\author{
Rishik Vashisht ${ }^{1}$, Saeid Mirzai ${ }^{2}$, Christine Koval ${ }^{3}$, Abhijit Duggal $^{4}$
}

\begin{abstract}
Aim: Viral causes of acute respiratory distress syndrome (ARDS) are mostly limited to influenza A; however, adenovirus has been emerging as a cause of fulminant ARDS with a high mortality rate and no consensus on its management. Here we present a series of five patients with confirmed adenovirus infection treated for ARDS at our quaternary referral institution.

Materials and methods: All patients were above 18 years old, had confirmed adenovirus infection, and were treated for acute hypoxic respiratory failure requiring mechanical ventilation in our medical intensive care unit (MICU). Demographic and clinical data were collected and analyzed. Results: Among these patients, the median age was 28 years, median BMI $28 \mathrm{~kg} / \mathrm{m}^{2}$, median sequential organ failure assessment (SOFA) score 9 , and median acute physiology and chronic health evaluation (APACHE) III score 74. All patients received lung-protective mechanical ventilation with high positive end-expiratory pressure and low plateau pressures. Three patients developed severe ARDS, two received prone position ventilation, and one was placed on extracorporeal membrane oxygenation. The median duration of mechanical ventilation, MICU length of stay, and hospital length of stay were 24,19 , and 27 days, respectively. One out of five patients died in our study.

Conclusion: The mortality rate for adenovirus-associated pneumonia in the literature is estimated to be $40 \%$ in those requiring mechanical ventilation. The lower mortality at our institution could be attributed to the use of standardized protocols, which include low tidal volume ventilation, early use of neuromuscular blockade, targeting low plateau pressures, conservative fluid management, and comfort and familiarity with the use of adjunctive and rescue therapies. We recommend testing for adenovirus as part of a routine respiratory viral panel in ARDS patients, and if tested positive, transfer to tertiary or quaternary centers with the experience and rescue modalities needed to manage complicated ARDS patients.
\end{abstract}

Keywords: Acute respiratory distress syndrome, Adenovirus, Cidofovir, Extracorporeal membrane oxygenation, Mechanical ventilation Indian Journal of Critical Care Medicine (2020): 10.5005/jp-journals-10071-23428

\section{To the Editor}

Acute respiratory distress syndrome (ARDS) is a rapid inflammatory lung injury with a high mortality rate ranging from 34.9 to $46.1 \% .^{1}$ Influenza and rhinovirus have been most commonly detected in viral pneumonias followed by parainfluenza, adenovirus, respiratory syncytial virus, coronavirus, and human metapneumovirus. ${ }^{2}$ Except for influenza $A$, most of the viral pneumonias rarely progress to ARDS. ${ }^{2}$ Adenovirus infections are usually self-limiting; however, dissemination and fatal pneumonias have been reported in a small subset of patients. Adenovirusassociated pneumonia can be fulminant with a mortality rate of approximately $40 \%$ in patients requiring mechanical ventilation. ${ }^{3}$

We report a case series of five patients with confirmed adenovirus infection who were treated for acute hypoxic respiratory failure requiring mechanical ventilation in the medical intensive care unit (MICU) at our quaternary referral institution between December 2015 and December 2016. Approval was obtained from the institutional review board of our hospital (Cleveland Clinic, Cleveland, $\mathrm{OH}$ ). Four patients were transferred to our MICU from outside hospitals because of rapid respiratory deterioration. The initial presentation in all patients was related to hypoxemic respiratory failure with eventual shock that required support with vasopressors. Three of the patients developed acute kidney injury requiring dialysis. Among the patients who developed ARDS, the course of the disease was fulminant in three of them and they developed rapidly worsening hypoxemia that progressed to severe ARDS.

All patients were placed on a lung-protective strategy $\left(V_{t}\right)$ and required support with high positive end expiratory pressure (PEEP) targeting a plateau pressure of $<30 \mathrm{~cm} \mathrm{H}_{2} \mathrm{O}$. Among the three patients who developed severe ARDS, all of them required deep sedation (RASS

\footnotetext{
1,4 Department of Critical Care, Respiratory Institute, Cleveland Clinic, Cleveland, Ohio, USA

${ }^{2}$ Department of Medicine, Alabama College of Osteopathic Medicine Dothan, Alabama, USA

${ }^{3}$ Department of Infectious Disease, Cleveland Clinic, Cleveland, Ohio, USA

Corresponding Author: Rishik Vashisht, Department of Critical Care, Respiratory Institute, Cleveland Clinic, Cleveland, Ohio, USA, Phone: +1-610-800-9629, e-mail: vashisr@ccf.org

How to cite this article: Vashisht R, Mirzai S, Koval C, Duggal A. Adenovirus-associated Acute Respiratory Distress Syndrome: Need for a Protocol-based Approach. Indian J Crit Care Med 2020;24(5):367-368.

Source of support: Nil

Conflict of interest: None
}

of -5$)$, and neuromuscular blocking agents were used to facilitate mechanical ventilation due to extremely poor lung compliance. Due to ongoing refractory hypoxemia, two patients underwent prone position ventilation (PPV) and one had to be placed on extracorporeal membrane oxygenation (ECMO). The median duration of mechanical ventilation was 24 days [IQR (15-31)], median ICU length of stay 19 days [IQR (19-31)], and median hospital length of stay 27 days [IQR (24-39)]. One patient died due to cardiac arrest as a result of refractory acidosis. Among the survivors, there was significant morbidity with three patients needing inpatient rehabilitation and one needing transfer to a skilled nursing facility after ECMO decannulation.

Acute respiratory distress syndrome in the context of isolated viral illness can be underrecognized, undertreated, 
and can consequently be associated with high mortality. This underrecognition leads to less optimal use of lung-protective ventilation strategies such as low $V_{t}$ ventilation, higher PEEP, and low plateau pressure. Less experienced centers may also be reluctant to use adjuncts to conventional lung-protective strategies such as the early use of neuromuscular blockade, PPV, strict adherence to conservative fluid management, and the initiation of ECMO in cases with refractory hypoxemia, all of which have shown to improve outcomes in large randomized controlled trials. A 2009 randomized controlled trial by the CESAR trial collaboration (efficacy and economic assessment of conventional ventilatory support vs extracorporeal membrane oxygenation for severe adult respiratory failure) showed improved survival without disability when a patient with ARDS was transferred to a center with an ECMO-based management protocol. ${ }^{4}$ Even patients who were not in the ECMO group showed improvements in mortality with the use of a standardized protocol for disease management in a highly experienced center. ${ }^{4}$ Similarly, Kahn et al. in 2006 showed that an increase in hospital volume was associated with improved survival in patients receiving mechanical ventilation. ${ }^{5}$

Our study additionally highlights that patients with adenovirusassociated ARDS have a protracted phase of acute, severe illness. They tend to have significant ICU and hospital lengths of stay with intensive rehabilitation requirements. We recommend a conservative approach in terms of family discussions about the withdrawal of care in such patients. We also recommend testing for adenovirus as part of a routine respiratory viral panel, and if tested positive, these patients should be transferred to tertiary or quaternary centers with specialized skill sets and experience in managing complicated ARDS patients with the resources for various rescue modalities.

\section{References}

1. Bellani G, Laffey JG, Pham T, Fan E, Brochard L, Esteban A, et al. Epidemiology, patterns of care, and mortality for patients with acute respiratory distress syndrome in intensive care units in 50 countries. JAMA 2016;315(8):788-800. DOI: 10.1001/jama.2016.0291.

2. Luyt CE, Combes A, Nieszkowska A, Trouillet JL, Chastre J. Viral infections in the ICU. Curr Opin Crit Care 2008;14(5):605-608. DOI: 10.1097/MCC.0b013e32830f1e12.

3. Tan D, Zhu H, Fu Y, Tong F, Yao D, Walline J, et al. Severe community-acquired pneumonia caused by human adenovirus in immunocompetent adults: a multicenter case series. PLoS ONE 2016;11(3):e0151199. DOI: 10.1371/journal.pone.0151199.

4. Peek GJ, Mugford M, Tiruvoipati R. CESAR trial collaboration. Efficacy and economic assessment of conventional ventilatory support versus extracorporeal membrane oxygenation for severe adult respiratory failure (CESAR): a multicentre randomised controlled trial. Lancet 2009;374(9698):1351-1363. DOI: 10.1016/S0140-6736(09)61069-2.

5. Kahn JM, Goss CH, Heagerty PJ, Kramer AA, O'Brien CR, Rubenfeld $G D$. Hospital volume and the outcomes of mechanical ventilation. N Engl J Med 2006;355(1):41-50. DOI: 10.1056/NEJMsa053993. 